\title{
Ureteral fibroepithelial polyp diagnosed preoperatively on virtual CT ureteroscopy
}

Chen-Pin Chou, ${ }^{1,3,4}$ Tony T. Wu, ${ }^{2,4}$ Robin B. Levenson, ${ }^{3}$ Jer-Shyung Huang, ${ }^{1,4}$ Huay-Ben $\operatorname{Pan}^{1,4}$

${ }^{1}$ Department of Radiology, Kaohsiung Veterans General Hospital, 386 Ta-Chung 1st Rd., Kaohsiung 813, Taipei, Taiwan, ROC

${ }^{2}$ Department of Urology, Kaohsiung Veterans General Hospital, Taipei, Taiwan, ROC

${ }^{3}$ Department of Radiology, UMass Memorial Medical Centre and University of Massachusetts Medical School, Worcester, MA 01655, USA

${ }^{4}$ National Yang-Ming University, School of Medicine, Taipei, Taiwan, ROC

Erratum to: Abdom Imaging DOI: $10.1007 / \mathrm{s} 00261-006-9084-1$

The published article contains errors uncorrected by the publisher at proof.

In the article opening page:

The author's name is Robin B. Levensen

It should read:

Robin B. Levenson

The online version of the original article can be found at doi: $10.1007 / \mathrm{s} 00261-006-9084-1$

Correspondence to: Chen-Pin Chou; email: r2207759@ms19.hinet.net 